\title{
New interpretation of arterial stiffening due to cigarette smoking using a structurally motivated constitutive model
}

\author{
Enevoldsen, Marie Sand; Henneberg, Kaj-Åge; Jensen, Jørgen Arendt; Lönn, L; Humphrey, J.D.
}

Published in:

Journal of Biomechanics

Link to article, DOI:

10.1016/j.jbiomech.2011.01.032

Publication date:

2011

Link back to DTU Orbit

Citation (APA):

Enevoldsen, M. S., Henneberg, K-Å., Jensen, J. A., Lönn, L., \& Humphrey, J. D. (2011). New interpretation of arterial stiffening due to cigarette smoking using a structurally motivated constitutive model. Journal of Biomechanics, 44(6), 1209-1211. https://doi.org/10.1016/j.jbiomech.2011.01.032

\section{General rights}

Copyright and moral rights for the publications made accessible in the public portal are retained by the authors and/or other copyright owners and it is a condition of accessing publications that users recognise and abide by the legal requirements associated with these rights.

- Users may download and print one copy of any publication from the public portal for the purpose of private study or research.

- You may not further distribute the material or use it for any profit-making activity or commercial gain

- You may freely distribute the URL identifying the publication in the public portal 


\title{
New interpretation of arterial stiffening due to cigarette smoking using a structurally motivated constitutive model
}

\author{
M.S. Enevoldsen ${ }^{\text {a,* }}$, K.-A. Henneberg ${ }^{\text {a }}$, J.A. Jensen ${ }^{a}$, L. Lönn ${ }^{\text {b }}$, J.D. Humphrey ${ }^{c}$ \\ ${ }^{a}$ Department of Electrical Engineering, Technical University of Denmark, Oersteds Plads, Building 349, DK-2800 Kongens Lyngby, Denmark \\ ${ }^{\mathrm{b}}$ Department of Radiology, Rigshospitalet, University of Copenhagen, Copenhagen, Denmark \\ ' Department of Biomedical Engineering, Yale University, New Haven, CT, USA
}

\section{A R T I C L E I N F O}

Article history:

Accepted 27 January 2011

\section{Keywords:}

Elastin

Collagen

Anisotropy

Stress

Biomechanics

\begin{abstract}
A B S T R A C T
Cigarette smoking is the leading self-inflicted risk factor for cardiovascular diseases; it causes arterial stiffening with serious sequelea including atherosclerosis and abdominal aortic aneurysms. This work presents a new interpretation of arterial stiffening caused by smoking based on data published for rat pulmonary arteries. A structurally motivated "four fiber family" constitutive relation was used to fit the available biaxial data and associated best-fit values of material parameters were estimated using multivariate nonlinear regression. Results suggested that arterial stiffening caused by smoking was reflected by consistent increase in an elastin-associated parameter and moreover by marked increase in the collagen-associated parameters. That is, we suggest that arterial stiffening due to cigarette smoking appears to be isotropic, which may allow simpler phenomenological models to capture these effects using a single stiffening parameter similar to the approach in isotropic continuum damage mechanics. There is a pressing need, however, for more detailed histological information coupled with more complete biaxial mechanical data for a broader range of systemic arteries.
\end{abstract}

(c) 2011 Elsevier Ltd. All rights reserved.

\section{Introduction}

Cardiovascular disease is a leading cause of death worldwide and cigarette smoking is one of six major associated modifiable risk factors (Doll et al., 2004). For example, production of nitric oxide by the endothelium, which mediates vasodilatation in response to altered hemodynamics, is reduced in both active and passive smokers (Barua et al., 2001). Hence, endothelial dysfunction caused by smoking can diminish the dilatory capacity of arteries, which yields a stiffer wall, as well as promote thrombus formation and inflammation within the wall. Although these and similarly detrimental consequences of smoking have long been recognized, detailed experimental data on associated histo-mechanical changes to arteries are sparse. There is, therefore, a pressing need for more experimental data, a better understanding of effects on extracellular matrix (ECM) constituents, and associated constitutive modeling of bulk changes in mechanical behavior. The goal of this paper is to gain a better understanding of consequences of smoking on arterial properties and ECM constituents based on changes in material parameters in a proven structurally motivated constitutive relation for arteries. This goal was achieved by re-interpreting experimental data from main pulmonary arteries in rats (Liu and Fung, 1993b) using a

\footnotetext{
* Corresponding author. Tel.: +45 45253705; fax: +45 45880117

E-mail address: mse@elektro.dtu.dk (M.S. Enevoldsen).
}

new "four fiber family" constitutive relation for stress (cf. Ferruzzi et al., 2010).

\section{Methods}

\subsection{Experimental data}

Inflation-extension data were inferred from Liu and Fung (1993b); see the original paper for details. Briefly, 28 initially three-month old Sprague-Dawley rats were exposed to either room air or cigarette smoke for two or three months, thus yielding four groups of seven rats each: Group 1 represented two-month controls, Group 2 the two-month smokers, Group 3 the three-month controls, and Group 4 the three-month smokers. The main pulmonary artery was excised for inflation-extension testing and data were analyzed in terms of the second PiolaKirchhoff stress (S) and Green strain (E). To facilitate use of the four fiber family constitutive relation, the biaxial data from their constitutive relation $\mathbf{S}=\hat{\mathbf{S}}(\mathbf{E})$ was regenerated in terms of components of the Cauchy stress $\mathbf{t}=\left(\mathbf{F} \cdot \mathbf{S} \cdot \mathbf{F}^{T}\right) / \mathrm{J}$, where $\mathbf{F}$ is the deformation gradient and $J$ its determinant (accomplished assuming no rotations, that is, $\mathbf{R}=\mathbf{I}$ and $\mathbf{V} \equiv \mathbf{U}$ using the polar decomposition theorem $\mathbf{F}=\mathbf{R} \cdot \mathbf{U}=\mathbf{V} \cdot \mathbf{R}$ where $\mathbf{U}$ and $\mathbf{V}$ are the right and left stretch tensors, respectively) and recalling that $\mathbf{E}=\left(\mathbf{F}^{T} \cdot \mathbf{F}-\mathbf{I}\right) / 2$. Note, too, that the constitutive relation in Liu and Fung (1993b) contained reference Green strains, values of which were not given. These reference strains were assumed to be $E_{\theta \theta}^{*}=0.182$ and $E_{z z}^{*}=0.274$ based on similar data by Debes and Fung (1995).

\subsection{Constitutive framework}

The primary load bearing constituents of the ECM in passive pulmonary arteries were assumed to be an amorphous matrix dominated by elastin and four 
families of locally parallel collagen fibers (axial, circumferential, and symmetric diagonal). The strain energy density function was thus assumed to have the form Ferruzzi et al. (2010),

$W\left(\mathbf{C}, \mathbf{M}^{(k)}\right)=\frac{c}{2}\left(I_{C}-3\right)+\sum_{k=1}^{4} \frac{c_{1}^{(k)}}{4 c_{2}^{(k)}}\left\{\exp \left[c_{2}^{(k)}\left(I V_{C}^{(k)}-1\right)^{2}\right]-1\right\}$,

where $c, c_{1}^{(k)}, c_{2}^{(k)}$ are material parameters, $I_{C}$ is the first invariant of the right Cauchy-Green tensor $\mathbf{C}=\mathbf{F}^{\mathrm{T}} \cdot \mathbf{F}$, and $I V_{C}^{(k)}=\mathbf{M}^{(k)} \cdot \mathbf{C M}^{(k)}$ is the fourth invariant of $\mathbf{C}$. Assuming incompressibility and plane stress $\left(t_{r r}=0\right)$, the theoretically predicted non-zero stress components of stress are

$$
\begin{aligned}
t_{\theta \theta}= & c\left(\lambda_{\theta}^{2}-\frac{1}{\lambda_{\theta}^{2} \lambda_{z}^{2}}\right)+c_{1}^{(2)}\left(\lambda_{\theta}^{2}-1\right) \exp \left[c_{2}^{(2)}\left(\lambda_{\theta}^{2}-1\right)^{2}\right] \lambda_{\theta}^{2} \\
& +2 c_{1}^{(3,4)}\left(I V_{C}^{(3,4)}-1\right) \exp \left[c_{2}^{(3,4)}\left(I V_{C}^{(3,4)}-1\right)^{2}\right] \lambda_{\theta}^{2} \sin ^{2}\left(\alpha_{0}\right) \\
t_{z z}= & c\left(\lambda_{z}^{2}-\frac{1}{\lambda_{\theta}^{2} \lambda_{z}^{2}}\right)+c_{1}^{(1)}\left(\lambda_{z}^{2}-1\right) \exp \left[c_{2}^{(1)}\left(\lambda_{z}^{2}-1\right)^{2}\right] \lambda_{z}^{2} \\
& +2 c_{1}^{(3,4)}\left(I V_{C}^{(3,4)}-1\right) \exp \left[c_{2}^{(3,4)}\left(I V_{C}^{(3,4)}-1\right)^{2}\right] \lambda_{z}^{2} \cos ^{2}\left(\alpha_{0}\right)
\end{aligned}
$$

where superscripts 1,2 , and 3,4 denote axial, circumferential, and symmetric diagonal fibers, respectively, and $\alpha_{0}$ denotes the orientation of the diagonal fibers relative to the axial direction in the reference configuration. This constitutive relation was selected because it has faithfully captured differential changes in the contribution of elastic fibers and fibrillar collagens to load bearing by the arterial wall in two cases of genetic mutations to elastin-associated proteins as well as in both aging of and the development of aneurysms in the human abdominal aorta (Eberth et al., 2009; Ferruzzi et al., 2010; Wan et al., 2010).

\subsection{Parameter estimation}

Best-fit values of the eight material parameters were determined via nonlinear regression by minimizing differences between the theoretically predicted and experimentally measured Cauchy stresses in circumferential and axial directions (using the Optimization Toolbox in MATLAB ${ }^{\mathrm{TM}} 7.8 .0$ (R2009a) with the build-in function Isqnonlin; see Ferruzzi et al., 2010 for details). Specifically, the objective function to be minimized was

$e(\boldsymbol{\beta})=\sum_{i=1}^{n}\left\{\left[t_{z z}^{\mathrm{th}}\left(\lambda_{z}, \lambda_{\theta} ; \boldsymbol{\beta}\right)_{i}-\left(t_{z z}^{\exp }\right)_{i}\right]^{2}+\left[t_{\theta \theta}^{\mathrm{th}}\left(\lambda_{z}, \lambda_{\theta} ; \boldsymbol{\beta}\right)_{i}-\left(t_{\theta \theta}^{\exp }\right)_{i}\right]^{2}\right\}$,

where $\boldsymbol{\beta}$ is the vector containing the eight material/structural parameters and $n$ is the number of data points $(n \gg 8)$. The most representative set of best-fit parameters was chosen as the mean of each parameter based on 100 successive estimations using randomly generated initial guesses that respected the physical constraint that all parameters remain non-negative. Note that Ferruzzi and colleagues have shown via an extensive non-parametric statistical analysis that results from such estimations tend to be robust, yielding unique fixed point estimates.

\section{Results}

An illustrative fit to data shows that the model captured well the biaxial data (Fig. 1). In general, the Cauchy stress was higher in the circumferential direction compared to the axial direction, and slightly higher in the older rats. More importantly, smoking caused significant stiffening in both age groups - stresses were generally three times greater in smokers compared to controls when evaluated at a common, upper-level deformation. The effect of smoking on the best-fit values of material parameters in the four fiber family constitutive relation was a marked increase in the value of all parameters, but particularly those associated with collagen $\left(c_{1}^{(k)}, c_{2}^{(k)}\right)$, see Table 1 .

\section{Discussion}

This re-interpretation of existing data on effects of smoking on arterial stiffness confirms that smoking accelerates stiffening of pulmonary arteries (cf. Doonan et al., 2010), but provides increased
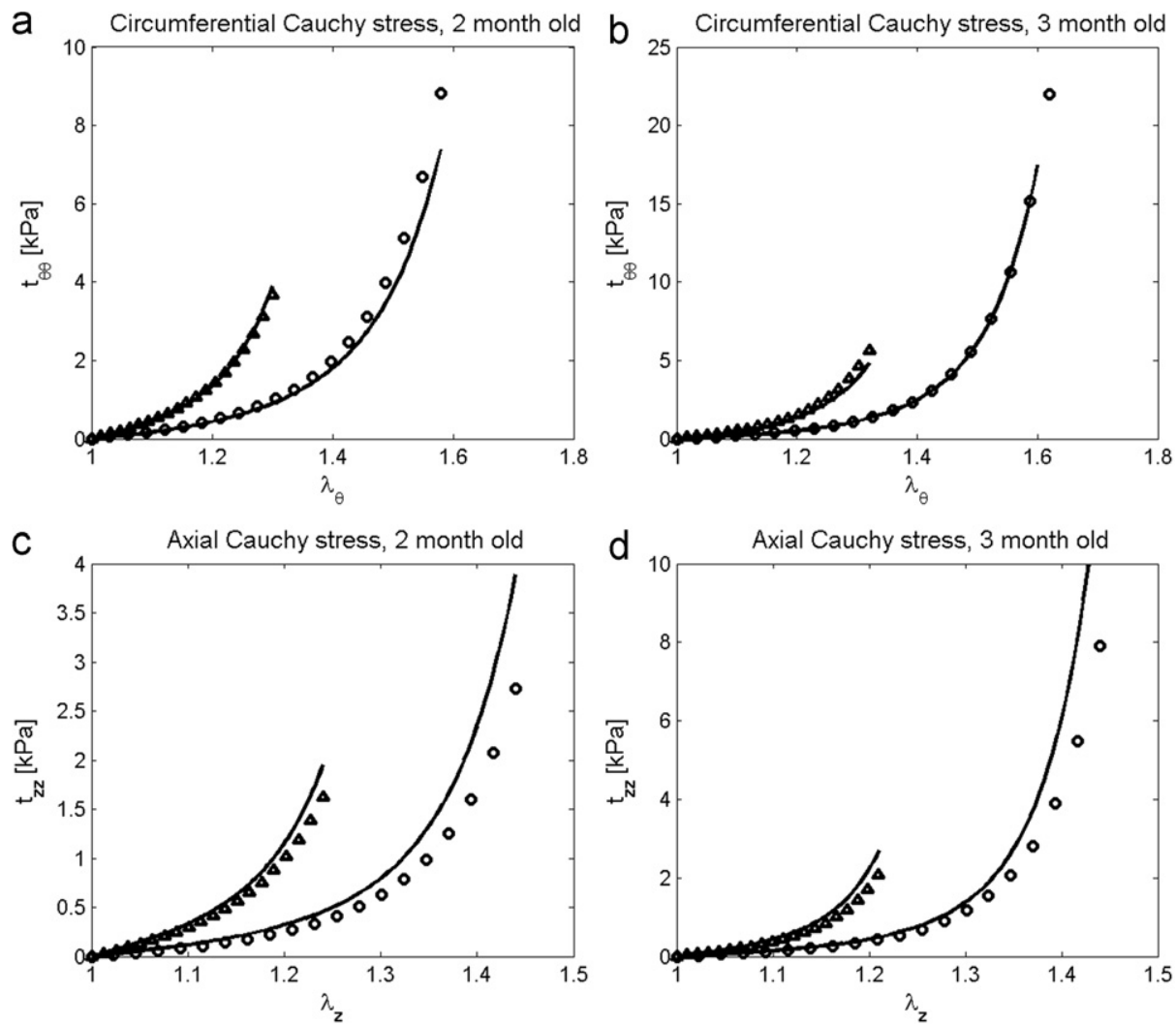

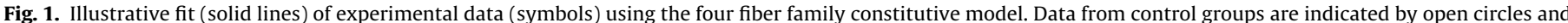

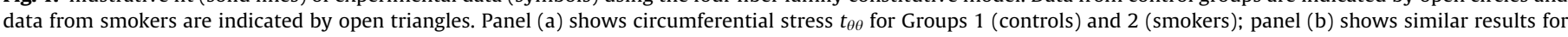

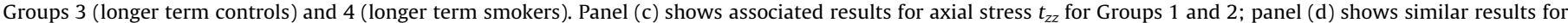
Groups 3 and 4 . 
Table 1

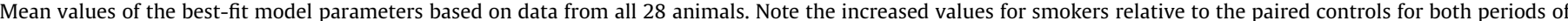

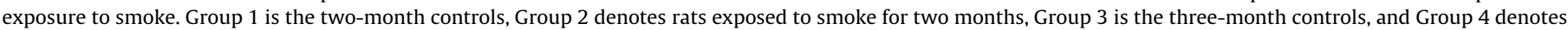

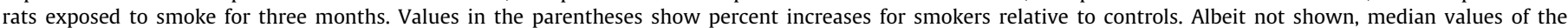
parameters represented much better the pooled data for each group than did mean values.

\begin{tabular}{|c|c|c|c|c|c|c|c|c|}
\hline Group & $c(\mathrm{kPa})$ & $c_{1}^{1}(\mathrm{kPa})$ & $c_{2}^{1}$ & $c_{1}^{2}(\mathrm{kPa})$ & $c_{2}^{2}$ & $c_{1}^{3,4}(\mathrm{kPa})$ & $c_{2}^{3,4}$ & $\alpha_{0}$ (deg.) \\
\hline 1 & 0.072 & 0.13 & 1.72 & 0.411 & 0.564 & 0.131 & 0.932 & 43.5 \\
\hline 2 & $0.142(+97 \%)$ & $0.424(+226 \%)$ & $3.39(+97.1 \%)$ & $0.915(+123 \%)$ & $1.77(+214 \%)$ & $0.44(+236 \%)$ & $2.16(+132 \%)$ & $48.8(+12.2 \%)$ \\
\hline 3 & 0.079 & 0.143 & 2.81 & 0.545 & 0.814 & 0.13 & 1.57 & 26.5 \\
\hline 4 & $0.139(+75.9 \%)$ & $0.454(+217 \%)$ & $6.28(+123 \%)$ & $1.16(+113 \%)$ & $1.62(+99.0 \%)$ & $0.408(+214 \%)$ & $3.56(+127 \%)$ & $38.1(+43.8 \%)$ \\
\hline
\end{tabular}

insight into this process. Liu and Fung (1993a) showed that cigarette smoking stimulates an increase in the volume fractions of both elastin and collagen, together with a decrease in smooth muscle cells in pulmonary rat arteries. The present structurally motivated constitutive relation similarly suggested an increased stiffness due to increases in both the elastin related (through the parameter $c$ ) and collagen related (through the parameters $c_{1}^{(k)}$ and $c_{2}^{(k)}$ ) terms, but the effect due to the latter appeared more marked. That is, the elastin related parameter increased by $\sim 85 \%$ whereas parameters associated with collagen in general increased at least two fold, thus suggesting overall nearly isotropic stiffening dominated by collagen.

The material symmetry associated with stiffening due to smoking has not received prior attention in the literature, but increased research on this aspect of stiffening may help increase our understanding of associations between smoking and cardiovascular disease and could simplify the phenomenological modeling that continues to dominate vascular mechanics. For example, one may be able to use a concept similar to that in isotropic damage mechanics to model progressive changes in arterial stiffening due to the duration or intensity of smoking. Toward this end, however, there is a pressing need for more data, particularly on the time course of long-term effects of smoking. We also focused on pulmonary arteries simply because the associated data were the most complete. Consequently, there is also a need for similar data on systemic arteries, especially carotids, cerebrals, coronaries, and aorta which are susceptible to smoking related diseases. Note, therefore, that we attempted a similar analysis of data from Cox et al. (1984), who reported findings on longer term effects of smoking on carotids and femorals from dogs. The associated parameter estimation was deemed unreliable, however, due to their focus on pressure-diameter data alone and too few data points within the systemic pressure range. There is, therefore, a critical need for more complete biaxial data, which in turn will permit more extensive evaluations of the associated constitutive behaviors. Consistent with other modeling studies (cf. Baek et al., 2007), note that we assumed an additive decomposition of the energy stored in the elastin-dominated and collagen-dominated portions of the ECM and further that the elastin responds isotropically (i.e., a neo-Hookean model). Clearly, interactions among the many different proteins that constitute the arterial wall should be expected, and models will need to account for these interactions once they are understood better. Although isotropy of the stress response of elastin has been questioned recently (e.g., Zou and Zhang, 2009; Lillie et al., 2010; Sokolis, 2010), most think that this possible anisotropy manifests primarily at low strains. Because of our ultimate interest in behaviors at physiological levels of strain, and consistent with findings by Gundiah et al. (2009) that neoHookean behavior dominates the stress response of elastin, we retained this assumption herein. Although such an assumption has allowed altered contributions of elastin to wall mechanics to be captured previously (Eberth et al., 2009; Ferruzzi et al., 2010; Wan et al., 2010), this as well as each aspect of the constitutive modeling needs to be considered more carefully as more detailed histological and mechanical data on the effect of smoking become available.
In summary, the increased stiffening of arteries due to smoking appears to result from concomitant changes to elastin and collagen, though effects on collagen are more pronounced perhaps because of its intrinsically higher stiffness. These effects appeared to occur nearly isotropically, which could simplify future analysis of the more complete data that are needed.

\section{Conflict of interest statement}

There is no conflict of interest.

\section{Acknowledgments}

We thank Mr. Jacopo Ferruzzi for providing custom software for the parameter estimation. This work was conducted in the Continuum Biomechanics Laboratory at Texas A\&M University and supported, in part, by project 55562 at Technical University of Denmark, Radiometer Medical ApS., Danish Ministry of Science Technology and Innovation, and NIH R01 HL086418.

\section{References}

Baek, S., Gleason, R.L., Rajagopal, K.R., Humphrey, J.D., 2007. Theory of small on large large: potential utility in computations of fluid-solid interactions in arteries. Computer Methods in Applied Mechanics and Engineering 196, 3070-3078.

Barua, R.S., Ambrose, J.A., Eales-Reynolds, L.J., DeVoe, M.C., Zervas, J.G., Saha, D.C. 2001. Dysfunctional endothelial nitric oxide biosynthesis in healthy smokers with impaired endothelium-dependent vasodilatation. Circulation 104, 1905-1910.

Cox, R.H., Tulenko, T., Santamore, W.P., 1984. Effects of chronic cigarette smoking on canine arteries. American Journal of Physiology 246, H97-H103.

Debes, J.C., Fung, Y.C., 1995. Biaxial mechanics of excised canine pulmonary arteries. Heart and Circular Physiology 38, H433-H442.

Doll, R., Peto, R., Boreham, J., Sutherland, I., 2004. Mortality in relation to smoking: 50 years' observations on male British doctors. British Medical Journal 328, 1519.

Doonan, R.J., Hausvater, A., Scallan, C., Mikhailidis, D.P., Pilote, L., Daskalopoulou, S.S. 2010. The effect of smoking on arterial stiffness. Hypertension Research, 1-13.

Eberth, J.F., Taucer, A.I., Wilson, K., Humphrey, J.D., 2009. Mechanics of carotid arteries in a mouse model of Marfans syndrome. Annals of Biomedical Engineering 37, 1093-1104.

Ferruzzi, J., Vorp, D.A., Humphrey, J.D., 2010. On constitutive descriptors of the biaxial mechanical behaviour of human abdominal aorta and aneurysms. Journal of the Royal Society, Interface (Pub ahead of print).

Gundiah, N., Ratcliffe, M.B., Pruitt, L.A., 2009. The biomechanics of arterial elastin. Journal of the Mechanical Behavior of Biomedical Materials 2, 288-296.

Lillie, M.A., Shadwick, R.E., Gosline, J.M., 2010. Mechanical anisotropy of inflated elastic tissue from the pig aorta. Journal of Biomechanics 43, 2070-2078.

Liu, S.Q., Fung, Y.C., 1993a. Changes in the structure and mechanical properties of pulmonary arteries in rats exposed to cigarette smoke. The American review of respiratory disease $148,768-777$.

Liu, S.Q., Fung, Y.C., 1993b. Material coefficients of the strain energy function of pulmonary arteries in normal and cigarette-exposed rats. Journal of Biomechanics 26, 1261-1269.

Sokolis, D., 2010. A passive strain energy function for elastic and muscular arteries: correlation of material parameters with histological data. Medical and Biological Engineering and Computing 48, 507-518.

Wan, W., Yanagisawa, H., Gleason, R.L., 2010. Biomechanical and microstructural properties of common carotid arteries from fibulin-5 null mice. Annals of Biomedical Engineering 38, 3605-3617.

Zou, Y., Zhang, Y., 2009. An experimental and theoretical study on the anisotropy of elastin network. Annals of Biomedical Engineering 37, 1572-1583. 